\title{
Survival results with the Use of Chemoradiotherapy in the Treatment of Locally Advanced Non-Small Cell Lung Cancer
}

\author{
Hanan Gamal-Eldeen Mostafa \\ Department of Clinical Oncology, Faculty of Medicine Assiut University, Assiut, Egypt
}

\begin{abstract}
Background: A combination of chemotherapy (CT) and radiotherapy (RT) is the treatment base for locally advanced non-small cell lung cancer (NSCLC). The aim of this work was to compare the survival impact of concomitant versus sequential CT and RT for inoperable (stage III) NSCLC.

Patients and Methods: The patients presented with stage III NSCLC, divided into 2 groups, group A treated with cisplatin $60 \mathrm{mg} / \mathrm{m}^{2}$, day1 plus etoposide $100 \mathrm{mg} / \mathrm{mg}^{2} /$ day, days $1-3$ and after 3 weeks RT (60 Gy in 30 fractions) started with weekly cisplatin $20 \mathrm{mg} / \mathrm{m}^{2}$ for 6 weeks followed by 3 cycles of the same CT after the end of RT. Group B treated with the same CT for 3 cycles followed by the same RT protocol, and then 3 cycles after the end of radiotherapy.

Results: The overall response rates were $55 \%$ in the concurrent group and $40 \%$ in the sequential group. Median survival was significantly longer in the concurrent group 20 months versus 12 months in the sequential group $12(P<0.001)$. Progression free survival was significantly longer in the concurrent group 15 months versus 9 months in the sequential group $(P<0.001)$. Toxicity was more frequent in the concurrent than in the sequential group with a significant greater incidence of leucopenia, esophagitis, nausea and vomiting $(P<0.001)$.

Conclusion: In this study population, concurrent CRT demonstrated significant benefit in terms of overall and progression-free survival over sequential CRT but at the cost of increased toxicity.
\end{abstract}

Key words: Non-small cell lung cancer, stage III, chemoradiotherapy, cisplatin and etoposide.

Corresponding Author: Hanan Gamal El-deen Mostafa

E-mail: mostafahanan36@yahoo.com

\section{INTRODUCTION}

Lung cancer is the most common and deadly tumor worldwide and approximately 1-3 million patients a year die of it $^{1}$. Non-small cell lung cancer accounts for $85 \%$ of all new cases diagnosed. Most patients are diagnosed with a non-resectable disease and around $40 \%$ in advanced stage ${ }^{2}$. Locally advanced disease (stageIII) is defined as a tumor that exceeds the structures of the lung itself, but without clinical evidence of distant spreading. In the past, radiotherapy was considered the standard therapy in stage IIIA and IIIB, but demonstrated very low survival, poor local control and early development of distant disease. Patients with inoperable stage III treated only with thoracic radiotherapy experienced a median survival of 9-11 months, 2-year survival of $10-20 \%$ and 3 -year survival of $5-10 \%{ }^{3}$. Cure is unlikely in those patients with locally advanced non-small cell lung cancer (NSCLC) who do not receive radical surgery; patients who receive chemotherapy and concomitant radiotherapy have a 3-year survival of approximately $27 \%{ }^{4}$. In 1995 , a meta-analysis based on individual data from 3,033 patients showed that the combining chemotherapy and radiotherapy gave statistically significant benefit. This difference was greater in those trials that had used platinum treatment in favor of combined chemotherapy and radiotherapy treatment ${ }^{5}$.

Once the benefit of using chemotherapy and radiotherapy was established, the best sequences of treatment became the great unknown. A meta-analysis of 12 clinical trials with 1,921 patients at various stages analyzed the role of chemotherapy based on cisplatin associated with radiotherapy and concluded that the addition of cisplatin to radiotherapy improves survival with absolute benefit of $4 \%$ at 2 years $(P<0.02)$ and the combination of cisplatin and etoposide is more effective than cisplatin alone ${ }^{6}$.

It should be noted that toxicity increases with concurrent treatment, particularly due to grade 3-4 esophagitis. Patients who are undergo concurrent therapy regimens need to be selected using strict criteria to exclude those with weight loss or extensive exposure of lungs to radiotherapy ${ }^{7}$. 
A meta-analysis was published to clarify whether concurrent or sequential treatment is better. This included 1,205 patients with 6-years follow-up and demonstrated that concomitant treatment contributed absolute benefit overall survival at 5 years of $4.5 \%$ over sequential treatment, but at the cost of increasing toxicity in the form of grade 3-4 esophagitis ${ }^{8}$.

The aim was to compare between sequential and concomitant chemotherapy and radiotherapy in patients with advanced inoperable non-small cell lung cancer.

\section{PATIENTS AND METHODS}

This randomized single-blind phase III study was conducted in Clinical Oncology department, Assiut University Hospital. The study included 40 patients in each group, treated between January 2005 and September 2008. The study included patients with inoperable locally advanced (stage III) non-small cell lung cancer (staging of American Joint Committee on Cancer $6^{\text {th }}$ ed. 2002).

\section{Eligibility criteria:}

Inclusion criteria were, age 41-85 years, histopathologically proven, locally advanced unresectable non metastatic NSCLC, bidiminsionaly measurable disease, no previous treatment by radiotherapy or chemotherapy, Eastern Cooperative Oncology Group performance status $<2$, adequate hematologic, hepatic and renal functions.

Exclusion criteria included pleural or pericardial effusion, extensive vessel invasion, a diagnosis of smallcell lung cancer, previous treatment with chemotherapy or radiotherapy to lung cancer and impaired renal functions.

All eligible patients gave their informed consent prior to the inclusion in the study.

\section{Treatment plan:}

The sample size was calculated by using the formula of randomized controlled trials. Eighty eligible patients were divided into two groups each group included forty patients. Both groups were balanced in their clinicopathological features.

Group A included forty patients were treated with one cycle of cisplatin $60 \mathrm{mg} / \mathrm{m}^{2}$ (one hour i.v. infusion) on day 1 plus etoposide $100 \mathrm{mg} / \mathrm{m}^{2} /$ day (30 minutes i.v. infusion) on days 1-3. Radiotherapy was given concomitant with weekly cisplatin $20 \mathrm{mg} / \mathrm{m}^{2} 30$ minutes infusion on day one of the second cycle chemotherapy for 6 weeks. Radiotherapy was administered 2 hours after completion of chemoradiotherapy infusion. Three cycles of the same chemotherapy every 21 days were administered after completing radiotherapy. All patients received intravenous antiemetic on day 1 with pre- and postchemotherapy hydration.

Radiotherapy consisted of a total dose 60Gy in 30 fractions over 6 weeks (2Gy/fraction for 5 days, each week) using linear accelerator $6 \mathrm{MV}$, twodimensional radiotherapy. The radiation dose was administered to a planning target volume that included computed tomography visible primary tumor (prechemotherapy tumor volume) plus $1 \mathrm{~cm}$ margins in the transverse diameter and $1.5-2 \mathrm{~cm}$ margins in the vertical direction to account for daily setup errors and target motion, it also included elective irradiation of ipsilateral, contralateral hilar, mediastinal, subcarinal and occasionally supraclavicular areas in cases with upper lobe tumors involvement. This is phase I and 40 Gy was delivered by parallel opposing anteroposterior and posteranterior fields. Second phase radiotherapy was delivered to the primary tumor, ipsilateral hilar lymph nodes and $1 \mathrm{~cm}$ margin for organ motion during treatment with a direct lateral field had a gantry angle of $90^{\circ}$, weighted down to $50 \%$ to reduce irradiation to the opposite lung. Other fields included anterior $30^{\circ}$ wedge field with a gantry angle $\mathrm{O}^{\circ}$ and a posterior oblique wedge field had a gantry angle of $140^{\circ}$. Phase II delivered 20 Gy.

Group B included forty patients were treated with three cycle of cisplatin $60 \mathrm{mg} / \mathrm{m}^{2}$ on day 1 and etoposide $100 \mathrm{mg} / \mathrm{m}^{2}$ on days $1-3$ every 3 weeks followed by the same radiotherapy protocol. Three cycles of cisplatin and etoposide were given after the end of radiotherapy ${ }^{9}$.

\section{Patient evaluation:}

All patients underwent a full physical examination, assessment for hematology, renal functions and toxicity. This assessment was conducted every 3 weeks before chemotherapy and again before and after radiation therapy.

Chest x-ray, computed tomography (CT) scan including upper abdomen to assess liver and adrenal gland status were performed before treatment and was repeated 4 weeks after the end of treatment.

Bone scan was performed to all patients before treatment, but brain CT scan was performed if clinically indicated.

Tissue diagnosis was made using biopsy/ brush or bronchial aspirate obtained during fibreoptic bronchoscope. 


Vol. 8 | No. 3-4 $2012 \quad$ Hanan Gamal-Eldeen Mostafa

After completion of study treatment, patients were follow-up every month until disease progression, for a maximum of one year from the date of the last chemotherapy treatment.

Any treatment related side effects recorded and were followed up until resolution.

Tumor response was assessed according to World Health Organization (WHO) criteria $^{10}$. Progression free survival (PFS) was defined as the time from the start of chemotherapy until the date of progression. Overall survival (OS) was determined from the start of chemotherapy to the date of death or last follow-up.

Toxicity was assessed using National Cancer InstituteCommon Toxicity Criteria (NCI-CTC) version 2.0.

\section{Statistical methods:}

The primary end points of this study were overall survival (OS) and Progression-free survival (PFS). Secondary end points were response rate and toxicity evaluation of sequential and concomitant chemoradiotherapy. Chi-square test was used to compare differences in distribution of frequencies among various groups of response. P-value 0.05 was considered significant. Overall survival and progression-free survival were calculated using Kaplan-Meier method ${ }^{11}$.

\section{RESULTS}

Eighty patients were included between January 2005 and September 2008. Patients were divided into two groups, group A included forty patients who were treated by concomitant chemoradiotherapy and group B included forty patients who were treated by sequential chemoradiotherapy.

Table (1) shows the baseline patient and tumor characteristics. Both groups were well balanced in their clinico-pathological characteristics except for the percentage of males is significantly higher in group B. Most patients had stage IIIB and squamous cell carcinoma was the commonest histology $(55 \%$ \& $45 \%$ in group A\& B, respectively).

Table (2) shows the response rate at study end. There was no significant difference in response between the two groups but partial response was 55\% (22/40) in group A which was higher than the partial response rate in group B $40 \%(16 / 40)$. The percentage of patients with progressive disease was $7.5 \%(3 / 40)$ and $25 \%(10 / 40)$ in the group A and B respectively which indicate a better response to concomitant chemoradiotherapy.
Table (3) shows the difference in response rate in patients with stage IIIA and stage IIIB disease who received concomitant chemoradiotherapy and sequential chemoradiotherapy. The partial response rate is significantly higher in patients with stage III A who were treated with concurrent versus sequential chemoradiotherapy (59\% vs $41 \%$ ) and also in patients with stage IIIB ( $56 \%$ vs $44 \%$ ) $P<0.01$. The percentage of patients with progressive disease was significantly higher in patients treated with sequential than concomitant chemoradiotherapy, in stage IIIA $80 \%$ vs $20 \%$ and in stage IIIB $75 \%$ vs $25 \% P<$ O.O 1 .

Tables 4, 5 show the prognostic factors for response in the two groups. The presence of N2-N3 disease was found as a significant adverse prognostic factor.

Safety and toxicity are reported in Table (6), there was no grade 4 toxicity in both groups but grade 3 toxicity was significantly higher in patients treated with concomitant chemoradiotherapy than sequential. Leucopenia is the most common grade 3 hematological adverse events in both groups, occurring in $20 \%$ of patients treated with concomitant chemoradiotherpy whish was significantly higher than $5 \%$ of patients treated with sequential chemoradiotherapy $(P<0.001)$. Esophagitis, nausea and vomiting were the most frequent treatment related non-hematological toxicity; both were significantly higher in the concomitant chemoradiotherapy group.

Figure (1) shows OS in both groups. The median OS was 12 months (95\% CI: 10.67-13.37 months) for patients treated with sequential chemoradiotherapy (group B) and 20 months (95\% CI: 18.45-21.54 months) for patients treated with concomitant chemoradiotherapy (group A). The difference in OS was statistically significant $(P<0.001)$.

The 1- and 2-year survival rates were higher in the concurrent arm (68\% and $42 \%$, respectively) than in the sequential arm ( $48 \%$ and $24 \%$, respectively).

The 1- and 2-year progression-free survival was also higher in the concurrent arm $(60 \%$ and $22 \%$, respectively) than in the sequential arm (32\% and $8 \%$, respectively).

Figure (2) shows PFS in both groups. Progressionfree survival was 9 (95\%CI: 8.32-9.67 months) and 15 months (95\%CI: 12.52-17.47 months) in patients treated with sequential and concomitant chemoradiothrapy respectively. The difference was statistically significant $(P<0.001)$. 


\section{Survival Functions}

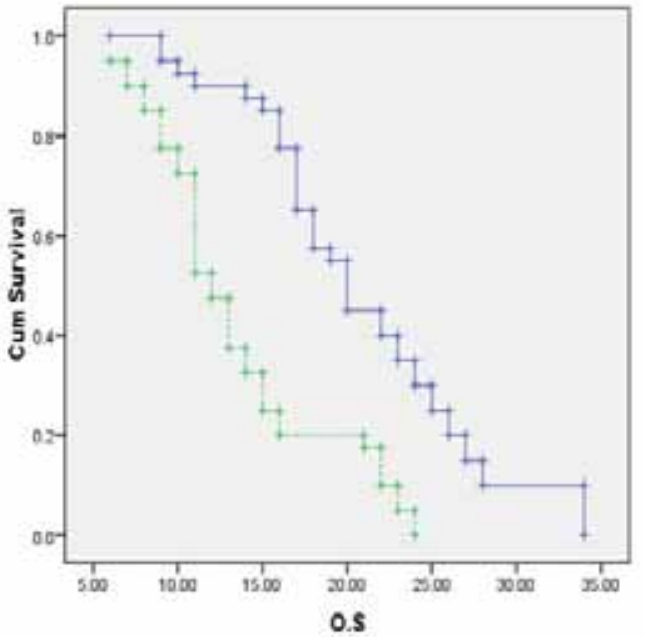

Figure 1: Overall survival (OS) of 40 patients with locally advanced non-small cell lung cancer (NSCLC) treated by concomitant chemoradiotherapy (C-CRT) group A (20 months, 95\% CI: $18.4521 .54-)$ versus OS of 40 patients with locally advanced NSCLC treated by sequential chemoradiotherapy (S-CRT) groupB (12 months, 95\% CI: 10.6713.32-) $P<0.001$. $+=$ censored patient

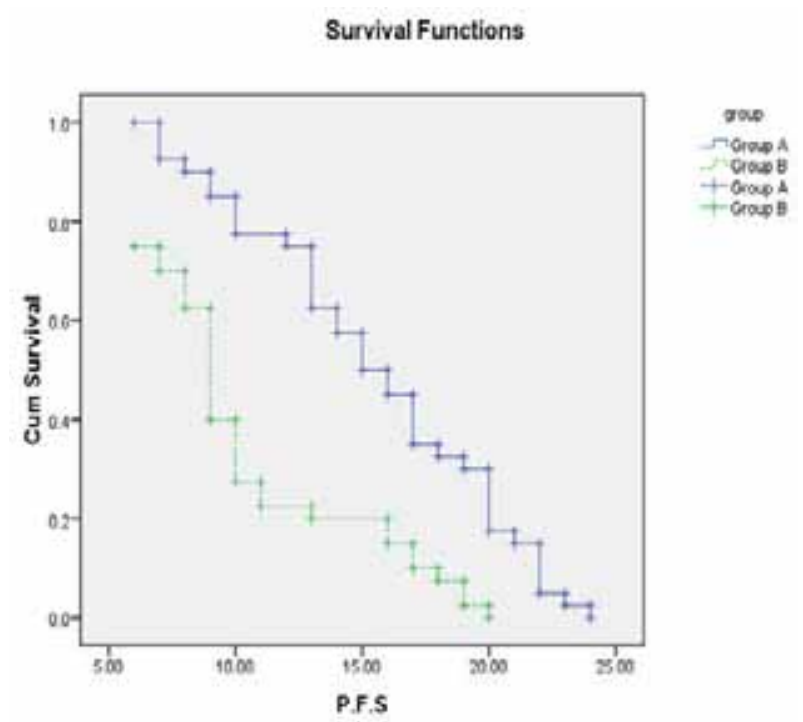

Figure 2: Progression-free survival (PFS) of 40 patients with locally advanced non-small cell lung cancer (NSCLC) treated by concomitant chemoradiotherapy (C-CRT) group A (15 months, CI: 12.5217.47-) versus PFS of 40 patients with locally advanced NSCLC treated by sequential chemoradiotherapy (S-CRT) group B (9 months, CI: 8.329.67-) $P<0.001$. $+=$ censored patients

Table 1: Clinico-pathological features of both groups.

\begin{tabular}{|c|c|c|c|}
\hline Group & $\begin{array}{c}\text { Group A } \\
40 \text { Patients }\end{array}$ & $\begin{array}{l}\text { Group B } \\
40 \text { Patients }\end{array}$ & $P$. Value \\
\hline \multicolumn{4}{|l|}{ Age (years) } \\
\hline Mean /SD & $60.17 \pm 10.85$ & $59.67 \pm 11.50$ & \multirow{3}{*}{0.372} \\
\hline Range & $41-84$ & $42.0-84.0$ & \\
\hline Median & 59.0 & 62.0 & \\
\hline \multicolumn{4}{|c|}{ ECOG Performance status: No (\%) } \\
\hline 0 & 24. $(60 \%)$ & $26(65 \%)$ & \multirow{2}{*}{0.409} \\
\hline 1 & $16(40 \%)$ & $14(35 \%)$ & \\
\hline \multicolumn{4}{|l|}{ Sex: No (\%) } \\
\hline Male & $30(75 \%)$ & $22(55 \%)$ & \multirow{2}{*}{$0.05^{*}$} \\
\hline Female & $10(25 \%)$ & $18(45 \%)$ & \\
\hline \multicolumn{4}{|l|}{ Histopathology: No (\%) } \\
\hline Adencarcinoma & $11(27.5 \%)$ & $11(27.5 \%)$ & \multirow{4}{*}{0.494} \\
\hline Large cell carcinoma & $2(5 \%)$ & $1(2.5 \%)$ & \\
\hline Squamous cell carcinoma & $22(55 \%)$ & $18(45 \%)$ & \\
\hline Others & $5(12.5 \%)$ & $10(25.0 \%)$ & \\
\hline \multicolumn{4}{|l|}{ Disease stage: No (\%) } \\
\hline III A & $19(47.5 \%)$ & $18(45.0 \%)$ & \multirow{2}{*}{0.50} \\
\hline III B & $21(52.5 \%)$ & $22(55.0 \%)$ & \\
\hline \multicolumn{4}{|l|}{ Nodal Status: } \\
\hline $\mathrm{N} 0-\mathrm{N} 1$ & $15(37.5 \%)$ & $13(32.5 \%)$ & \multirow{2}{*}{0.3} \\
\hline $\mathrm{N} 2-\mathrm{N} 3$ & $25(62.5 \%)$ & $27(67.5 \%)$ & \\
\hline
\end{tabular}

ECOG: Eastern Cooperative Oncology Group

$*=$ statistically significant

Group A: concomitant chemoradiotherapy

Group B: sequential chemoradiotherapy 
Table 2: Response rates.

\begin{tabular}{lcccc}
\hline Treatment group & PR & So & PD \\
Concomitant chemoradiotherapy $(\mathrm{n}=40)$ & $22(55 \%)$ & $\begin{array}{c}\text { No (\%) } \\
\text { No (\%) }\end{array}$ & \multirow{2}{*}{0.274} \\
\cline { 1 - 2 } Sequential chemo- radiotherapy $(\mathrm{n}=40)$ & $16(40)$ & $15(37.5)$ & $3(7.5)$ & $10(25)$ \\
\hline
\end{tabular}

PR: Partial response, SD: Stable disease, PD: Progressive disease

Table 3: Response rate in stage IIIA and IIIB according to the treatment.

\begin{tabular}{|c|c|c|c|c|}
\hline \multirow{2}{*}{ Treatment } & \multicolumn{4}{|c|}{ Response (\%) } \\
\hline & PR & SD & PD & $P$. Value \\
\hline \multicolumn{5}{|l|}{ C-CRT } \\
\hline Stage III A ( $\mathrm{n}=19)$ & $13(59)$ & $5(50)$ & $1(20)$ & \\
\hline S-CRT & & & & $0.01^{*}$ \\
\hline Stage III A(n=18) & $9(41)$ & $5(50)$ & $4(80)$ & \\
\hline \multicolumn{5}{|l|}{ C- RT } \\
\hline Stage IIIB $(n=21)$ & $9(56)$ & $10(53)$ & $2(25)$ & \\
\hline S-CRT & & & & $0.01 *$ \\
\hline Stage IIIB $(n=22)$ & $7(44)$ & $9(47)$ & $6(75)$ & \\
\hline
\end{tabular}

PR: Partial response, SD: Stable disease, PR: Progressive disease

C-CRT: Concomitant chemoradiotherapy, S-CRT: Sequential chemoradiotherapy

$*=$ statistically significant

Table 4: Prognostic factors in Group A (concomitant chemoradiotherapy).

\begin{tabular}{|c|c|c|c|c|c|}
\hline Item & & $P R$ “n=22” & SD "n=15" & PD “n=3” & $P$-value \\
\hline \multicolumn{6}{|c|}{ ECOG Performance status: } \\
\hline • & 1 & $7(31.8 \%)$ & $8(53.3 \%)$ & $1(33.3 \%)$ & \multirow{2}{*}{0.411} \\
\hline • & 0 & $15(37.5 \%)$ & $7(46.7 \%)$ & $2(66.7 \%)$ & \\
\hline \multicolumn{6}{|c|}{ Histology: } \\
\hline • & Adencarcinoma & $12(54.5 \%)$ & $9(60.0 \%)$ & $1(33.3 \%)$ & \multirow{4}{*}{0.912} \\
\hline • & Large cell carcinoma & $6(27.3 \%)$ & $4(26.7 \%)$ & $1(33.3 \%)$ & \\
\hline • & Squamous cell carcinoma & $1(4.5 \%)$ & $1(6.7 \%)$ & -- & \\
\hline • & Others & $3(13.6 \%)$ & $1(6.7 \%)$ & $1(33.3 \%)$ & \\
\hline \multicolumn{6}{|c|}{ Lymph node status: } \\
\hline • & N0-N1 & $15(68.18 \%)$ & -- & -- & \multirow{2}{*}{$0.03 *$} \\
\hline - & N2-N3 & $7(31.82 \%)$ & $15(100 \%)$ & $3(100 \%)$ & \\
\hline
\end{tabular}

ECOG: Eastern Cooperative Oncology Group

PR: partial response, SD: stable disease, PD: progressive disease

*: significant $\mathrm{P}$ value 
Table 5: Prognostic factors in Group B (sequential chemoradiotherapy).

\begin{tabular}{|c|c|c|c|c|}
\hline Item & PR "n=16" & SD "n=14" & PD "n=10" & $P$-value \\
\hline \multicolumn{5}{|l|}{ ECOG Performance status: } \\
\hline - 1 & $7(43.8 \%)$ & $4(28.6 \%)$ & $3(30.0 \%)$ & \multirow{2}{*}{0.637} \\
\hline 0 & $9(56.2 \%)$ & $10(71.4 \%)$ & $7(70.0 \%)$ & \\
\hline \multicolumn{5}{|l|}{ Histology: } \\
\hline - $\quad$ Adencarcinoma & $6(37.5 \%)$ & $7(50.0 \%)$ & $5(50.0 \%)$ & \multirow{4}{*}{0.396} \\
\hline Large cell carcinoma & $7(43.8 \%)$ & $3(21.4 \%)$ & $1(10.0 \%)$ & \\
\hline Squamous cell carcinoma & ----- & $1(7.1 \%)$ & -- & \\
\hline Others & $3(18.8 \%)$ & $3(21.4 \%)$ & $4(40.0 \%)$ & \\
\hline \multicolumn{5}{|l|}{ Lymph node status: } \\
\hline N0-N1 & $11(68.75 \%)$ & $2(14.29 \%)$ & -- & \multirow{2}{*}{$0.03 *$} \\
\hline $\mathrm{N} 2-\mathrm{N} 3$ & $5(31.25 \%)$ & $12(85.71 \%)$ & $10(100 \%)$ & \\
\hline
\end{tabular}

ECOG: Eastern Cooperative Oncology Group

PR: partial response, SD: stable disease, PD: progressive disease

*: significant $\mathrm{P}$ value

Table 6: Hematologic and nonhematologic toxicity: NCI-CTC grade III.

\begin{tabular}{lcc}
\hline & & Treatment \\
\cline { 2 - 3 } Toxicity & $\begin{array}{c}\text { C-CRT } \\
(\mathbf{n = 4 0 )}\end{array}$ & $\begin{array}{c}\text { S-CRT } \\
(\mathbf{n}=\mathbf{4 0})\end{array}$ \\
\hline Hematological: no (\%) & & - \\
Anemia & $2(5)$ & $2(5)$ \\
Leucopenia & $8(20)$ & 0.001 \\
\hline Nonhematological: no (\%) & & $4(10)$ \\
Nausea \& Vomiting & $6(15)$ & $2(5)$ \\
Esophagitis & $8(20)$ & 0.001 \\
\hline
\end{tabular}

NCI-CTC: National Cancer Institute- Common Toxicity Criteria

C-CRT: Concomitant chemoradiotherapy, S-CRT: Sequential chemoradiotherapy

\section{DISCUSSION}

Despite intensive investigation, the prognosis for patients with lung cancer, up to $87 \%$ of whom have nonsmall cell lung cancer (NSCLC) at diagnosis, remains poor, with an estimate 5-year survival rate of only $15 \%{ }^{12}$.

The standard treatment of locally advanced unresectable NSCLC is combined chemotherapy and thoracic radiation, based on the results of several randomized phase III trials ${ }^{13,14}$ subsequent trials have demonstrated the superiority of concurrent chemotherapy and radiotherapy over sequential approach at the expense of increased toxicity, in particular sever esophagitis ${ }^{15,16}$.

Concurrent chemoradiotherapy improves overall survival (OS) of patients with locally advanced NSCLC compared with sequential chemoradiotherapy. Platinumbased polychemotherapy is considered the standard treatment. The most active combination in this situation is cisplatin-etoposide which provides a median survival of 23 months (overall survival at 3 years of $26.1 \%$, progression-free survival around 10 months $)^{17,18}$.

In the present study, the partial response rate was not significantly higher in patients treated with concurrent versus sequential chemoradiotherapy (55\%vs. $40 \%$,) but a significant response rate was noted to concomitant chemoradiotherapy than sequential in stage III A and B. This is in agreement with the results of the study done by Fournel et al. ${ }^{19}$ who reported a better response rate of $(54 \%$ vs. $49 \%)$ in the concurrent versus sequential chemoradiotherapy. Similar to the current study findings, stage IIIA patients gained particular benefit from concomitant chemoradiotherapy by achieving an overall response rate almost $20 \%$ higher than those treated with radiotherapy alone ${ }^{20}$.

These data indicate that concurrent chemoradiotherapy is helpful in improving response and survival than sequential one; this is also seen in previous studies done by Zatloukal et al. ${ }^{16}$ and Wang et al. ${ }^{21}$. 


Vol. 8 | No. 3-4 $2012 \quad$ Hanan Gamal-Eldeen Mostafa

The presence of N2-N3 disease was found to be an adverse prognostic factor in the present study (Table 4,5) but in the study done by Saynak et al..$^{9}$ non-epidermoid histology and Karnofsky Performance Status less than 70 were found as additional prognostic factors.

Median survival times in the current study were 20 months versus 12 months $(P<0.001)$ for patients treated with concurrent versus sequential chemoradiotherapy which is similar to the results of the study done by Crvenkova et al. ${ }^{22}$ who reported a median survival 13 months for patients treated with sequential chemoradiotherapy and 22 months for patients treated with concurrent chemoradiotherapy $(P<0.001)$. Conversely, in a study done by Saynak et al. ${ }^{9}$ a similar median survival was reported in both groups (14.5 vs. 14.6 months) for patients treated with sequential and concomitant chemoradiotherapy respectively, mostly due to the inclusion of patients with stage III B only in his study.

The 1- and 2-year survival rates were $68 \%$ and $42 \%$ in the concurrent group and $48 \%$ and $24 \%$ in the sequential group was noted in the current study which is in agreement with the statistical significant difference in 1 - and 2-year survival rates of $73.6 \%$ and $39.7 \%$ in the concurrent group and $45.4 \%$ and $13.7 \%$ in the sequential group in a study done by Crvenkova et al. ${ }^{22}$ Another study done by fournel et al. ${ }^{19}$ also reported a better 2-year survival rates in the concurrent arm than sequential $\operatorname{arm}(39 \%$ vs $26 \%$, respectively).

In the present study, Progression-free survival (PFS) was significantly higher in patients treated with concurrent than sequential chemoradiotherapy (15 vs. 9 months $P<0.001)$. This is in agreement with the significant difference in DFS in the study done by Crvenkova et al. ${ }^{22}$ (17 vs. 9 months $\left.P<0.001\right)$.

The 1-year progression-free survival rate was $60 \%$ which is lower than the 1-year PFS results reported by Wang et al. ${ }^{21}(75 \%)$, the difference in local control rates may be due to the use of three dimensional conformal radiotherapy in his study.

Both treatments were well tolerated; no grade 4 toxicity was reported in both groups. Notably, little toxicity reported with an increased incidence significantly, especially acute esophagitis and leucopenia, in patients treated with concomitant than sequential chemoradiotherapy. These results were in agreement with results reported by Crevenkova et $a l .{ }^{22}$ but grade 3 esophagitis in his study was a reason for radiotherapy interruption during conformal threedimensional radiotherapy may be due to the use of high dose chemotherapy concomitantly with radiotherapy.
In an attempt to improve loco-regional control in stage III A-B NSCLC, a three armed randomized trial comparing accelerated radiotherapy or concurrent daily or weekly chemotherapy with conventional radiotherapy was tested by Nyman et al. ${ }^{23}$.

Treatment results are quite equal by intensifying the locoregional treatment either by accelerated fractionated radiotherapy or daily or weekly concurrent chemoradiotherpy both in term of survival, toxicity and quality of life.

A phase III randomized study comparing concomitant radiochemotherapy as induction versus consolidation treatment in patients with locally advanced unresectable NSCLC done by Berghmans et al. ${ }^{24}$. They concluded that consolidation chemoradiotherapy seems less toxic with a better observed response rates and survival.

It remains to be determined whether induction chemotherapy before concurrent chemoradiotherapy or concurrent chemoradiotherpy followed by consolidation chemotherapy is the most effective sequence, but the latter approach has produced a longer survival times. It is important to note that some patients with locally advanced NSCLC do not meet the tumor volume requirements when planning radiotherapy at baseline. Induction chemotherapy might potentially rescue some patients presenting with bulky disease if a policy of encompassing postchemotherapy tumor volume is adopted ${ }^{20}$.

\section{CONCLUSION}

In conclusion, the addition of chemotherapy to radiation concomitantly prolongs survival than sequential therapy in locally advanced non-small cell lung cancer patients with acceptable adverse event profiles.

\section{REFERENCES}

1. Parkin DM, Bray F, Ferlay J, Pisani P. Global cancer statistics, 2002. CA Cancer J.Clin. 2005;55(2):74-108.

2. Ramalingam S, Belani C. Systemic chemotherapy for advanced non-small cell lung cancer: Recent advances and future directions. Oncologist 2008;13 Suppl 1:5-13.

3. Perez CA, Pajak TF, Rubin P, Simpson JR, Mohiuddin M, Brady LW, et al. Long-term observations of the patterns of failure in patients with unresectable non-oat cell carcinoma of the lung treated with definitive radiotherapy. Report by the Radiation Therapy Oncology Group. Cancer 1987;59(11):1874-81.

4. Hanna N, Neubauer M, Yiannoutsos C, McGarry R, Arseneau J, Ansari R, et al. Phase III study of cisplatin, etoposide and concurrent chest radiation with or without consolidation docetaxel in patients with inoperable stage III non-small-cell lung cancer: The Hoosier Oncology Group and U.S. Oncology. J.Clin.Oncol. 2008;26(35):5755-60. 
5. Chemotherapy in non-small cell lung cancer: A metaanalysis using updated data on individual patients from 52 randomised clinical trials. Non-small Cell Lung Cancer Collaborative Group. Br.Med.J. 1995;311(7010):899-909.

6. Aupérin A, Le Péchoux C, Pignon JP, Koning C, Jeremic B, Clamon G, et al. Concomitant radiochemotherapy based on platin compounds in patients with locally advanced non-small cell lung cancer (NSCLC): A meta-analysis of individual data from 1764 patients. Ann. Oncol. 2006;17(3):473-83.

7. Provencio M, Isla D, Sánchez A, Cantos B. Inoperable stage III non-small cell lung cancer: Current treatment and role of vinorelbine. J.Thorac.Dis. 2011;3(3):197-204.

8. Auperin A, Le Pechoux C, Rolland E, Curran WJ, Furuse $\mathrm{K}$, Fournel $\mathrm{P}$, et al. Meta-analysis of concomitant versus sequential radiochemotherapy in locally advanced non-small-cell lung cancer. J.Clin.Oncol. 2010; 28(13): 2181-90.

9. Saynak M, Aksu G, Fayda M, Kaytan E, Oral E, Gurocak S, et al. The results of concomitant and sequential chemoradiotherapy with cisplatin and etoposide in patients with locally advanced non-small cell lung cancer. J.Balkan Union Oncol. 2005;10(2):213-8.

10. Miller AB, Hoogstraten B, Staquet M, Winkler A. Reporting results of cancer treatment. Cancer 1981;47(1):207-14.

11. Kaplan EL, Meier P. Nonparametric estimation from incomplete observations. J.Am.Stat.Assoc. 1985;53(282):457-74.

12. Ries LAG, Eisner MP, Kosary CL, Hankey BF, Miller BA, Clegg L, et al. SEER Cancer Statistics Review 1975-2002 National Cancer Institute. Available: http://seer.cancer. gov/csr/1975_2002/,2005.

13. Dillman RO, Herndon J, Seagren SL. Improved survival in stage III non-small-cell lung cancer: Seven-year followup of cancer and leukemia Group B (CALGB) 8433 trial. J.Natl.Cancer Inst. 1996;88(17):1210-5.

14. Sause W, Kolesar P, Taylor SI, Johnson D, Livingston R, Komaki R, et al. Final results of phase III trial in regionally advanced unresectable non-small cell lung cancer: Radiation Therapy Oncology Group, Eastern Cooperative Oncology Group and Southwest Oncology Group. Chest 2000;117(2):358-64.

15. Curran WJ, Scott CB, Langer CJ. Long-term benefit is observed in a phase III comparison of sequential vs. concurrent chemo-radiation for patients with unresected stage III NSCLC RTOG 9410. Prog.Proc.Am.Soc.Clin. Oncol. 2003;22:621.
16. Zatloukal P, Petruzelka L, Zemanova M, Havel L, Janku F, Judas L, et al. Concurrent versus sequential chemoradiotherapy with cisplatin and vinorelbine in locally advanced non-small cell lung cancer: A randomized study. Lung Cancer 2004;46(1):87-98.

17. Albain KS, Swann RS, Rusch VW, Turrisi AT3, Shepherd FA, Smith C, et al. Radiotherapy plus chemotherapy with or without surgical resection for stage III non-small-cell lung cancer: A phase III randomised controlled trial. Lancet 2009;374(9687): 379-86

18. Hanna N, Neubauer M, Yiannoutsos C, McGarry R, Arseneau J, Ansari R, et al. Phase III study of cisplatin, etoposide and concurrent chest radiation with or without consolidation docetaxel in patients with inoperable stage III non-small-cell lung cancer: The Hoosier Oncology Group and U.S. Oncology. J.Clin.Oncol. 2008;26(35):5755-60.

19. Fournel P, Robinet G, Thomas P, Souquet PJ, Léna H, Vergnenégre A, et al. Randomized phase III trial of sequential chemoradiotherapy compared with concurrent chemoradiotherapy in locally advanced non-small-cell lung cancer: Groupe Lyon-Saint-Etienne d'Oncologie Thoracique-Groupe Français de Pneumo-Cancérologie NPC 95-01 Study. J.Clin.Oncol. 2005;23(25):5910-7.

20. Scagliotti GV, Szczesna A, Ramlau R, Cardenal F, Mattson K, Van Zandwijk N, et al. Docetaxel-based induction therapy prior to radiotherapy with or without docetaxel for non-small-cell lung cancer. Br.J.Cancer 2006;94(10):1375-82.

21. Wang J, Pang QS, Wang P, Wang J, Wang W. [Weekly paclitaxel and carboplatin with concurrent three dimensional conformal radiotherapy for locally advanced non small cell lung cancer]. Zhonghua Zhong Liu Za Zhi 2007;29(10):769-72.

22. Crvenkova S, Krstevska V. Sequential chemoradiotherapy compared with concurrent chemoradiotherapy in locally advanced non-small cell lung cancer: Our experience. Prilozi 2009;30(2):197-207.

23. Nyman J, Friesland S, Hallqvist A, Seke M, Bergstrom S, Thaning L, et al. How to improve loco-regional control in stages IIIa-b NSCLC?. Results of a three-armed randomized trial from the Swedish Lung Cancer Study Group. Lung Cancer 2009;65:62-7.

24. Berghmans T, Van Houtte P, Paesmans M, Giner V, Lecomte J, Koumakis G, et al. A phase III randomised study comparing concomitant radiochemotherapy as induction versus consolidation treatment in patients with locally advanced unresectable non-small cell lung cancer. Lung Cancer 2009;64(2):187-93. 